\title{
Subscription Dynamics and Competition in Communications Markets
}

\author{
Shaolei Ren, Jaeok Park, Mihaela van der Schaar \\ Electrical Engineering Department \\ University of California, Los Angeles \\ \{rsl,jaeok,mihaela\}@ee.ucla.edu
}

\begin{abstract}
Multiple technologies, possibly administered by different entities, are expected to coexist in the rapidly-expanding communications market. In order to understand the complex interactions between different technologies, it is of fundamental importance to understand how technologies affect the demand of users and competition between network service providers (NSPs). To this end, we analyze user subscription dynamics and competition between NSPs in a duopoly communications market. First, we investigate the impact of technologies on the users' dynamic subscription and show that, for any charged prices, the equilibrium point of the considered user subscription dynamics exists and is unique. Next, we derive a sufficient condition on the technologies of the NSPs that ensures the user subscription dynamics to reach the equilibrium point. Then, we model the NSP competition using a non-cooperative game, in which the two NSPs choose their market shares independently, and provide a sufficient condition that guarantees the existence of at least one pure Nash equilibrium in the market competition game.
\end{abstract}

\section{Categories and Subject Descriptors}

C.2.1 [Computer-Communication Networks]: Network Architecture and Design-Wireless Communications

\section{General Terms}

Design, Economics, Management

\section{INTRODUCTION}

Tremendous efforts have been dedicated in the past decade to enhancing the quality-of-service (QoS) of communications networks and expanding their network capacities. Nevertheless, it is the joint consideration of prices and QoS that determines the demand of users and the revenue of network service providers (NSPs). Moreover, investment in technologies by NSPs affects QoS and in turn the pricing schemes

Permission to make digital or hard copies of all or part of this work for personal or classroom use is granted without fee provided that copies are not made or distributed for profit or commercial advantage and that copies bear this notice and the full citation on the first page. To copy otherwise, to republish, to post on servers or to redistribute to lists, requires prior specific permission and/or a fee.

NetEcon'10, October 3, Vancouver, BC, Canada.

Copyright 2010 ACM ...\$10.00.

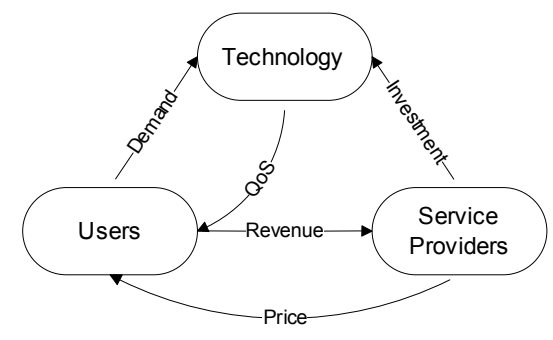

Figure 1: Interaction between technology, NSPs and users.

they use. Hence, technologies, NSPs and users are closely coupled and interact in a complex way (as illustrated in Fig. 1).

In this paper, we are interested in studying the interaction between technologies, the subscription decisions of users, and the pricing strategies of NSPs. We focus on a duopoly market, in which NSP $\mathcal{S}_{1}$ has sufficient resources and provides a constant QoS to its subscribers while NSP $\mathcal{S}_{2}$ provides each user with a QoS that depends on the number of subscribers. In particular, to take into account the QoS degradation when more users subscribe, the QoS of NSP $\mathcal{S}_{2}$ is modeled as a non-increasing function in terms of the number of subscribers. Given the provided QoS and charged prices, users dynamically select the NSP that yields a higher (non-negative) utility. We first show that, for any prices, the considered user subscription dynamics always admits a unique equilibrium point, at which no user wishes to change its subscription decision. We next obtain a sufficient condition that the QoS functions need to fulfill to guarantee the convergence of the user subscription dynamics. The analysis indicates that in order for the dynamics of user subscriptions to converge, the QoS provided by NSP $\mathcal{S}_{2}$ should not degrade too fast when more users subscribe to $\mathcal{S}_{2}$. Then, we analyze the competition between the two NSPs. Specifically, modeled as a strategic player in a non-cooperative game, each NSP aims to maximize its own revenue by selecting its own market share while regarding the market share of its competitor as fixed. This is in sharp contrast with the existing related literature which typically studies price competition among NSPs. For the formulated market share competition game, we derive a sufficient condition on the QoS function that guarantees the existence of at least one pure Nash equilibrium (NE).

In summary, the main contributions of this paper are as follows: (i) we study how different QoS functions (hence, 
different technologies) affect the user subscription and market competition by assuming a general QoS model for NSP $\mathcal{S}_{2}$ and a general distribution of the users' valuations of the QoS. This is unlike most of the existing literature where linear QoS functions and a uniform distribution are assumed; (ii) we find a sufficient condition which the QoS provided by NSP $\mathcal{S}_{2}$ needs to fulfill in order to guarantee the convergence of the user subscription dynamics; and (iii) we analyze the competition between two NSPs choosing their market shares strategically and characterize QoS functions that ensure the existence of a $\mathrm{NE}$ of the market share competition game.

The rest of this paper is organized as follows. Section II describes the model. In Section III, we study the user subscription dynamics and NSP competition in a duopoly communications market. An illustrative example is provided in Section IV and related work is reviewed in V. Finally, concluding remarks are offered in Section VI.

\section{SYSTEM MODEL}

We consider a communications market in which two NSPs, denoted by $\mathcal{S}_{1}$ and $\mathcal{S}_{2}$, operate. There is a continuum of users, as in [6], that can potentially subscribe to one of the NSPs for communications services. The continuum model approximates well the real user population if there are a sufficiently large number of users in the market so that each individual user is negligible [2]. As in [7][11], we assume throughout this paper that each user can subscribe to at most one NSP at any time instant. We also assume that NSP $\mathcal{S}_{1}$ has sufficient resources to provide a guaranteed level of QoS to all of its subscribers [7], whereas NSP $\mathcal{S}_{2}$ is resource-constrained and thus is prone to congestion among subscribers. ${ }^{1}$ In other words, the QoS provided by NSP $\mathcal{S}_{1}$ is the same regardless of the number of its subscribers, whereas the QoS provided by NSP $\mathcal{S}_{2}$ degrades with the number of its subscribers [1][2]. Let $\lambda_{i}$ be the fraction of users subscribing to NSP $\mathcal{S}_{i}$ for $i=1,2$. Then $\lambda_{1}$ and $\lambda_{2}$ satisfy $\lambda_{1}, \lambda_{2} \geq 0$ and $\lambda_{1}+\lambda_{2} \leq 1$. Also, let $q_{i}$ be the QoS provided by NSP $\mathcal{S}_{i}$ for $i=1,2$. Note that $q_{1}$ is independent of $\lambda_{1}$ while $q_{2}$ is non-increasing in $\lambda_{2}$. We use a function $g(\cdot)$ defined on $[0,1]$ to express the QoS provided by NSP $\mathcal{S}_{2}$ as $q_{2}=g\left(\lambda_{2}\right)$. For simplicity, we assume as in [4] that the cost of serving subscribers is fixed and smaller than the level that drives the NSPs out of the market so that we can use revenue maximization as the objective of the NSPs.

Users are heterogeneous in the sense that they may value the same level of QoS differently. Each user $k$ is characterized by a non-negative real number $\alpha_{k}$, which represents its valuation of QoS. Specifically, when user $k$ subscribes to NSP $\mathcal{S}_{i}$, its utility is given by

$$
u_{k, i}=\alpha_{k} q_{i}-p_{i},
$$

where $p_{i}$ is the subscription price charged by NSP $\mathcal{S}_{i}$, for $i=1,2 .{ }^{2}$ Users that do not subscribe to either of the two NSPs obtain zero utility. Note that in our model the NSPs are allowed to engage in neither QoS discrimination nor price

\footnotetext{
${ }^{1}$ An example that fits into our assumptions on NSPs is a cognitive radio network in which NSP $\mathcal{S}_{1}$ is a licensed operator serving primary users while NSP $\mathcal{S}_{2}$ is a spectrum broker serving secondary users. Another example is a market in which NSP $\mathcal{S}_{1}$ serves each user using a dedicated channel while NSP $\mathcal{S}_{2}$ has its users share its limited resources or capacity [2].

${ }^{2} \mathrm{~A}$ similar quasilinear utility model has been used in $[2][7][9]$.
}

discrimination. That is, all users subscribing to the same NSP receive the same QoS and pay the same subscription price [1][7].

Now, we impose assumptions on the QoS function of NSP $\mathcal{S}_{2}$, user subscription decisions, and the users' valuation of QoS as follows.

Assumption 1: $g(\cdot)$ is a non-increasing and continuously differentiable $^{3}$ function, and $0<g\left(\lambda_{2}\right)<q_{1}$ for all $\lambda_{2} \in$ $[0,1]$.

Assumption 2: Each user $k$ subscribes to NSP $\mathcal{S}_{i}$ if $u_{k, i}>$ $u_{k, j}$ and $u_{k, i} \geq 0$ for $i, j \in\{1,2\}$ and $i \neq j$. If $u_{k, 1}=u_{k, 2} \geq$ 0 , user $k$ subscribes to $\mathrm{NSP} \mathcal{S}_{1}{ }^{4}$

Assumption 3: The users' valuation of QoS follows a probability distribution whose probability density function (PDF) $f(\cdot)$ is strictly positive and continuous on $[0, \beta]$ for some $\beta>0$. For completeness of definition, we have $f(\alpha)=0$ for all $\alpha \notin[0, \beta]$. The cumulative density function (CDF) is given by $F(\alpha)=\int_{-\infty}^{\alpha} f(x) d x$ for $\alpha \in \mathbb{R}$.

We briefly discuss the above three assumptions. Assumption 1 captures the congestion effects that users experience when subscribing to resource-constrained NSP $\mathcal{S}_{2}$ (e.g., traffic congestion in [2][11]). The shape of the QoS function $g(\cdot)$ of NSP $\mathcal{S}_{2}$ is determined by various factors including the resource allocation scheme and the scheduling algorithm of NSP $\mathcal{S}_{2}$. Assumption 2 can be interpreted as a rational subscription decision. A rational user will subscribe to the NSP that provides a higher utility if at least one NSP provides a non-negative utility, and to neither NSP otherwise. Assumption 3 can be considered as an expression of user diversity in terms of the valuation of QoS. When the users' valuation of QoS is sufficiently diverse, its distribution can be described by a continuous positive PDF on a certain interval as in [2]. Note that the lower bound on the interval is set as zero to simplify the analysis, and this will be the case when there is enough diversity in the users' valuation of QoS so that there are non-subscribers for any positive price.

\section{COMPETITION IN COMMUNICATIONS MARKET}

In this section, we analyze user subscription dynamics and revenue maximization in a duopoly communications market where two competing NSPs $\mathcal{S}_{1}$ and $\mathcal{S}_{2}$ operate. We first analyze the equilibrium and convergence of user subscription dynamics, and then study revenue maximization in a scenario where the NSPs compete by choosing market shares.

\subsection{User Subscription Dynamics}

With the two NSPs operating in the market, each user has three possible choices at each time instant: subscribe to NSP $\mathcal{S}_{1}$, subscribe to NSP $\mathcal{S}_{2}$, and subscribe to neither. Since the QoS provided NSP $\mathcal{S}_{2}$ is varying with the fraction

${ }^{3}$ Since $g(\cdot)$ is defined on $[0,1]$, we use a one-sided limit to define the derivative of $g(\cdot)$ at 0 and 1 , i.e., $g^{\prime}(0)=\lim _{\lambda_{2} \rightarrow 0^{+}}\left[g\left(\lambda_{2}\right)-g(0)\right] /\left(\lambda_{2}-0\right)$ and $g^{\prime}(1)=$ $\lim _{\lambda_{2} \rightarrow 1^{-}}\left[g\left(\lambda_{2}\right)-g(1)\right] /\left(\lambda_{2}-1\right)$.

${ }^{4}$ Specifying an alternative tie-breaking rule (e.g., random selection between the two NSPs) in case of $u_{k, 1}=u_{k, 2} \geq 0$ will not affect the analysis of this paper, since the fraction of indifferent users is zero under Assumption 3 and thus the revenue of the NSPs is independent of the tie-breaking rule. A similar remark holds for the tie-breaking rule between subscribing and not subscribing in the case of $u_{k, i}=0 \geq u_{k, j}$ for $i, j=\{1,2\}$ such that $i \neq j$. 
of its subscribers, ${ }^{5}$ each user will form a belief, or expectation, on the QoS of NSP $\mathcal{S}_{2}$ when it makes a subscription decision. To describe the dynamics of user subscription, we construct and analyze a dynamic model which specifies how users form their beliefs and make decisions based on their beliefs. We consider a discrete-time model with time periods indexed $t=1,2, \ldots$. At each period $t$, user $k$ holds a belief or expectation on the QoS of NSP $\mathcal{S}_{2}$, denoted by $\tilde{g}_{k}\left(\lambda_{2}^{t}\right)$ where $\lambda_{2}^{t}$ is the fraction of subscribers at period $t$, and makes a subscription decision in a myopic way to maximize its expected utility in the current period. ${ }^{6}$ In particular, we specify that every user expects that the QoS in the current period is equal to that in the previous period. That is, we have $\tilde{g}_{k}\left(\lambda_{2}^{t}\right)=g\left(\lambda_{2}^{t-1}\right)$ for $t=1,2, \ldots$, where $\lambda_{2}^{0}$ is the initial fraction of subscribers. ${ }^{7}$ It is further assumed that, other than the subscription price, there is no cost involved in subscription decisions (e.g., initiation fees, termination fees, device prices) when users switch between NSP $\mathcal{S}_{1}$ and NSP $\mathcal{S}_{2}$ [6]. By Assumption 2, at period $t=1,2 \cdots$, user $k$ subscribes to NSP $\mathcal{S}_{1}$ if and only if

$$
\alpha_{k} q_{1}-p_{1} \geq \alpha_{k} g\left(\lambda_{2}^{t-1}\right)-p_{2} \text { and } \alpha_{k} q_{1}-p_{1} \geq 0,
$$

to NSP $\mathcal{S}_{2}$ if and only if

$$
\alpha_{k} g\left(\lambda_{2}^{t-1}\right)-p_{2}>\alpha_{k} q_{1}-p_{1} \text { and } \alpha_{k} g\left(\lambda_{2}^{t-1}\right)-p_{2} \geq 0,
$$

and to neither NSP if and only if

$$
\alpha_{k} q_{1}-p_{1}<0 \text { and } \alpha_{k} g\left(\lambda_{2}^{t-1}\right)-p_{2}<0 .
$$

Our model implies that, given the prices $\left(p_{1}, p_{2}\right)$, the user subscription dynamics in the duopoly market evolves following a sequence $\left\{\left(\lambda_{1}^{t}, \lambda_{2}^{t}\right)\right\}_{t=0}^{\infty}$ in $\Lambda=\left\{\left(\lambda_{1}, \lambda_{2}\right) \in \mathbb{R}_{+}^{2} \mid \lambda_{1}+\right.$ $\left.\lambda_{2} \leq 1\right\}$ generated by

$$
\begin{aligned}
& \lambda_{1}^{t}=h_{d, 1}\left(\lambda_{1}^{t-1}, \lambda_{2}^{t-1}\right) \triangleq 1-F\left(\frac{p_{1}-p_{2}}{q_{1}-g\left(\lambda_{2}^{t-1}\right)}\right) \\
& \lambda_{2}^{t}=h_{d, 2}\left(\lambda_{1}^{t-1}, \lambda_{2}^{t-1}\right) \triangleq F\left(\frac{p_{1}-p_{2}}{q_{1}-g\left(\lambda_{2}^{t-1}\right)}\right)-F\left(\frac{p_{2}}{g\left(\lambda_{2}^{t-1}\right)}\right)
\end{aligned}
$$

if $p_{1} / q_{1}>p_{2} / g\left(\lambda_{2}^{t-1}\right)$, and by

$$
\begin{aligned}
& \lambda_{1}^{t}=h_{d, 1}\left(\lambda_{1}^{t-1}, \lambda_{2}^{t-1}\right) \triangleq 1-F\left(\frac{p_{1}}{q_{1}}\right), \\
& \lambda_{2}^{t}=h_{d, 2}\left(\lambda_{1}^{t-1}, \lambda_{2}^{t-1}\right) \triangleq 0
\end{aligned}
$$

if $p_{1} / q_{1} \leq p_{2} / g\left(\lambda_{2}^{t-1}\right)$, for $t=1,2, \ldots$, starting from a given initial point $\left(\lambda_{1}^{0}, \lambda_{2}^{0}\right) \in \Lambda$. Note that there are two regimes of the user subscription dynamics in the duopoly market, and which regime governs the dynamics depends on the relative values of the prices per $Q o S$, i.e., $p_{1} / q_{1}$ and $p_{2} / g\left(\lambda_{2}^{t-1}\right)$.

Given the user subscription dynamics (5)-(8), we are interested in whether the fraction of subscribers will stabilize

\footnotetext{
5 "Fraction of subscribers" of an NSP is used throughout this paper to mean the proportion of users in the market that subscribe to this NSP.

${ }^{6}$ An example consistent with our subscription timing is a "Pay-As-You-Go" plan in which a subscribing user pays a fixed service charge for a unit of time (day, week, or month) and is free to quit its subscription at any time period, effective from the next time unit.

${ }^{7}$ This model of belief formation is called naive or static expectations in [3]. A similar dynamic model of belief formation and decision making has been extensively adopted in the existing literature, e.g., [6][7][11].
}

in the long run and, if so, to what value. As a first step, we define an equilibrium point of the user subscription dynamics as follows.

Definition 1: $\left(\lambda_{1}^{*}, \lambda_{2}^{*}\right)$ is an equilibrium point of the user subscription dynamics in the duopoly market if it satisfies

$$
h_{d, 1}\left(\lambda_{1}^{*}, \lambda_{2}^{*}\right)=\lambda_{1}^{*} \text { and } h_{d, 2}\left(\lambda_{1}^{*}, \lambda_{2}^{*}\right)=\lambda_{2}^{*} .
$$

We establish the existence and uniqueness of an equilibrium point and provide equations characterizing it in Proposition 1, whose proof can be found in [8].

Proposition 1. For any non-negative price pair $\left(p_{1}, p_{2}\right)$, there exists a unique equilibrium point $\left(\lambda_{1}^{*}, \lambda_{2}^{*}\right)$ of the user subscription dynamics in the duopoly market. Moreover, $\left(\lambda_{1}^{*}, \lambda_{2}^{*}\right)$ satisfies

$$
\begin{cases}\lambda_{1}^{*}=1-F\left(\frac{p_{1}}{q_{1}}\right), \lambda_{2}^{*}=0, & \text { if } \frac{p_{1}}{q_{1}} \leq \frac{p_{2}}{g(0)}, \\ \lambda_{1}^{*}=1-F\left(\theta_{1}^{*}\right), \lambda_{2}^{*}=F\left(\theta_{1}^{*}\right)-F\left(\theta_{2}^{*}\right), & \text { if } \frac{p_{1}}{q_{1}}>\frac{p_{2}}{g(0)},\end{cases}
$$

where $\theta_{1}^{*}=\left(p_{1}-p_{2}\right) /\left(q_{1}-g\left(\lambda_{2}^{*}\right)\right)$ and $\theta_{2}^{*}=p_{2} / g\left(\lambda_{2}^{*}\right)$.

Proposition 1 indicates that, given any prices $\left(p_{1}, p_{2}\right)$, the market shares of the two NSPs are uniquely determined when the fraction of users subscribing to each NSP no longer changes. It also shows that the structure of the equilibrium point depends on the relative values of $p_{1} / q_{1}$ and $p_{2} / g(0)$. Specifically, if the price per QoS of NSP $\mathcal{S}_{1}$ is always smaller than or equal to that of NSP $\mathcal{S}_{2}$, i.e., $p_{1} / q_{1} \leq p_{2} / g(0)$, then no users subscribe to NSP $\mathcal{S}_{2}$ at the equilibrium point. On the other hand, if NSP $\mathcal{S}_{2}$ offers a smaller price per QoS to its first subscriber than NSP $\mathcal{S}_{1}$ does, i.e., $p_{1} / q_{1}>p_{2} / g(0)$, then both NSP $\mathcal{S}_{1}$ and NSP $\mathcal{S}_{2}$ may attract a positive fraction of subscribers.

Our equilibrium analysis so far guarantees the existence of a unique stable point of the user subscription dynamics in the duopoly market. However, it does not discuss whether the unique stable point will be eventually reached. To answer this question, we turn to the analysis of the convergence properties of the user subscription dynamics. The convergence of the user subscription dynamics is not always guaranteed, especially when the QoS provided by NSP $\mathcal{S}_{2}$ degrades rapidly with respect to the fraction of subscribers. As a hypothetical example, suppose that only a small fraction of users subscribe to NSP $\mathcal{S}_{2}$ at period $t$ and each subscriber obtains a high QoS. In our model of belief formation, users expect that the QoS will remain high at period $t+1$, and thus a large fraction of users subscribe at period $t+1$, which will result in a low QoS at period $t+1$. This in turn will induce a small fraction of subscribers at period $t+2$. When the QoS of NSP $\mathcal{S}_{2}$ is very sensitive to the fraction of subscribers, the user subscription dynamics may oscillate around or diverge away from the equilibrium point and thus convergence may not be obtained. The following theorem, whose proof is deferred to [8], provides a sufficient condition under which the user subscription dynamics always converges.

THEOREM 1. For any non-negative price pair $\left(p_{1}, p_{2}\right)$, the user subscription dynamics specified by (5)-(8) converges to the unique equilibrium point starting from any initial point $\left(\lambda_{1}^{0}, \lambda_{2}^{0}\right) \in \Lambda$ if

$$
\max _{\lambda_{2} \in[0,1]}\left\{-\frac{g^{\prime}\left(\lambda_{2}\right)}{g\left(\lambda_{2}\right)} \cdot \frac{q_{1}}{q_{1}-g\left(\lambda_{2}\right)}\right\}<\frac{1}{K},
$$


where $K=\max _{\alpha \in[0, \beta]} f(\alpha) \alpha$.

The condition (11) in Theorem 1 is sufficient but not necessary for the convergence of the user subscription dynamics. In particular, we observe through numerical simulations that in some cases the user subscription dynamics converges for a wide range of prices although the condition (11) is violated (see [8] for more details). Nevertheless, the sufficient condition provides us with the insight that if QoS degradation is too fast (i.e., $-K g^{\prime}\left(\lambda_{2}\right) q_{1}$ is larger than $g\left(\lambda_{2}\right) \cdot\left[q_{1}-g\left(\lambda_{2}\right)\right]$ for some $\left.\lambda_{2} \in[0,1]\right)$, the dynamics may oscillate or diverge.

\subsection{Competition in a Duopoly Market}

We now study steady-state (or equilibrium) revenue maximization and competition in the duopoly market. ${ }^{8}$ In the economics literature, competition among a small number of firms has been analyzed using game theory, following largely two distinct approaches: Bertrand competition and Cournot competition. In Bertrand competition, firms choose prices independently while supplying quantities demanded at the chosen prices. On the other hand, in Cournot competition, firms choose quantities independently while prices are determined in the markets to equate demand with the chosen quantities. In the case of monopoly, whether the monopolist chooses the price or the quantity does not affect the outcome since there is a one-to-one relationship between the price and the quantity given a downward-sloping demand function. On the contrary, in the presence of strategic interaction, whether firms choose prices or quantities can affect the outcome significantly. For example, it is well-known that identical firms producing a homogeneous good obtain zero profit in the equilibrium of Bertrand competition while they obtain a positive profit in the equilibrium of Cournot competition, if they have a constant marginal cost of production and face a linear demand function.

Since the functions $\lambda_{i}^{*}\left(p_{1}, p_{2}\right), i=1,2$, are defined implicitly by (10), it is difficult to study the competition between the two NSPs using a Bertrand game (interested readers are referred to [8] for more details). We now consider Cournot competition between the two NSPs. Let $\lambda_{i} \in[0,1]$ be the market share chosen by NSP $\mathcal{S}_{i}$, for $i=1,2$. Suppose that $\lambda_{1}+\lambda_{2} \leq 1$ so that the chosen market shares are feasible. Let $p_{i}\left(\lambda_{1}, \lambda_{2}\right), i=1,2$, be the prices that clear the market, i.e., the prices that satisfy $\lambda_{i}=\lambda_{i}^{*}\left(p_{1}\left(\lambda_{1}, \lambda_{2}\right), p_{2}\left(\lambda_{1}, \lambda_{2}\right)\right)$ for $i=1,2$. Note first that, given a price pair $\left(p_{1}, p_{2}\right)$, if a user $k$ subscribes to NSP $\mathcal{S}_{1}$, i.e., $\alpha_{k} q_{1}-p_{1} \geq \alpha_{k} g\left(\lambda_{2}\right)-p_{2}$ and $\alpha_{k} q_{1}-p_{1} \geq 0$, then all the users whose valuation of QoS is larger than $\alpha_{k}$ also subscribe to NSP $\mathcal{S}_{1}$. Also, if a user $k$ subscribes to one of the NSPs, i.e., $\max \left\{\alpha_{k} q_{1}-p_{1}, \alpha_{k} g\left(\lambda_{2}\right)-\right.$ $\left.p_{2}\right\} \geq 0$, then all the users whose valuation of QoS is larger than $\alpha_{k}$ also subscribe to one of the NSPs. Therefore, realizing positive market shares $\lambda_{1}, \lambda_{2}>0$ requires two types of marginal users whose valuations of QoS are specified by $\alpha_{m, 1}$ and $\alpha_{m, 2}$ with $\alpha_{m, 1}>\alpha_{m, 2} . \alpha_{m, 1}$ is the valuation of QoS of a marginal user that is indifferent between subscribing to NSP $\mathcal{S}_{1}$ and NSP $\mathcal{S}_{2}$, while $\alpha_{m, 2}$ is the valuation of QoS of a marginal user that is indifferent between subscribing to NSP $\mathcal{S}_{2}$ and neither. The expressions for $\alpha_{m, 1}$ and $\alpha_{m, 2}$ that realizes $\left(\lambda_{1}, \lambda_{2}\right)$ such that $\lambda_{1}, \lambda_{2}>0$ and $\lambda_{1}+\lambda_{2} \leq 1$

${ }^{8}$ By focusing on equilibrium revenue, we implicitly assume that the unique equilibrium point is reached within a finite number of time periods. are given by

$$
\begin{aligned}
& \alpha_{m, 1}\left(\lambda_{1}, \lambda_{2}\right)=z_{1}\left(\lambda_{1}\right) \triangleq F^{-1}\left(1-\lambda_{1}\right), \\
& \alpha_{m, 2}\left(\lambda_{1}, \lambda_{2}\right)=z_{2}\left(\lambda_{1}, \lambda_{2}\right) \triangleq F^{-1}\left(1-\lambda_{1}-\lambda_{2}\right) .
\end{aligned}
$$

Also, by solving the indifference conditions, $\alpha_{m, 1} q_{1}-p_{1}=$ $\alpha_{m, 1} g\left(\lambda_{2}\right)-p_{2}$ and $\alpha_{m, 2} g\left(\lambda_{2}\right)-p_{2}=0$, we obtain a unique price pair that realizes $\left(\lambda_{1}, \lambda_{2}\right)$ such that $\lambda_{1}, \lambda_{2}>0$ and $\lambda_{1}+\lambda_{2} \leq 1$

$$
\begin{aligned}
p_{1}\left(\lambda_{1}, \lambda_{2}\right)= & F^{-1}\left(1-\lambda_{1}\right)\left[q_{1}-g\left(\lambda_{2}\right)\right] \\
& +F^{-1}\left(1-\lambda_{1}-\lambda_{2}\right) g\left(\lambda_{2}\right), \\
p_{2}\left(\lambda_{1}, \lambda_{2}\right)= & F^{-1}\left(1-\lambda_{1}-\lambda_{2}\right) g\left(\lambda_{2}\right) .
\end{aligned}
$$

Note that the expressions (12)-(15) are still valid even when $\lambda_{i}=0$ for some $i=1,2$, although uniqueness is no longer obtained. Hence, we can interpret $p_{i}(\cdot), i=1,2$, as a function defined on $\Lambda$ (an inverse demand function in economics terminology). Then the revenue of $\mathcal{S}_{i}$ when the NSPs choose $\left(\lambda_{1}, \lambda_{2}\right) \in \Lambda$ is given by $R_{i}\left(\lambda_{1}, \lambda_{2}\right)=\lambda_{i} p_{i}\left(\lambda_{1}, \lambda_{2}\right)$, for $i=1,2$. We define $R_{i}\left(\lambda_{1}, \lambda_{2}\right)=0, i=1,2$, if $\lambda_{1}+\lambda_{2}>1$, i.e., if the market shares chosen by the NSPs are infeasible. Cournot competition in the duopoly market can be formulated as a non-cooperative game specified by

$$
\mathcal{G}_{C}=\left\{\mathcal{S}_{i}, R_{i}\left(\lambda_{1}, \lambda_{2}\right), \lambda_{i} \in[0,1] \mid i=1,2\right\} .
$$

A market share pair $\left(\lambda_{1}^{* *}, \lambda_{2}^{* *}\right)$ is said to be a (pure) NE of $\mathcal{G}_{C}$ (or a Cournot equilibrium) if it satisfies

$$
R_{i}\left(\lambda_{i}^{* *}, \lambda_{-i}^{* *}\right) \geq R_{i}\left(\lambda_{i}, \lambda_{-i}^{* *}\right), \forall \lambda_{i} \in[0,1], \forall i=1,2 .
$$

Note that $(1,1)$ is a $\mathrm{NE}$ of $\mathcal{G}_{C}$, which yields zero profit to both NSPs. To eliminate this inefficient and counterintuitive equilibrium, we can restrict the strategy space of each NSP to $[0,1)$. Deleting 1 from the strategy space can also be justified by noting that $\lambda_{i}=1$ is a weakly dominated strategy for NSP $\mathcal{S}_{i}$, for $i=1,2$, since $R_{i}\left(1, \lambda_{-i}\right)=0 \leq R_{i}\left(\lambda_{i}, \lambda_{-i}\right)$ for all $\left(\lambda_{i}, \lambda_{-i}\right) \in[0,1]^{2}{ }^{9}$ We use $\tilde{\mathcal{G}}_{C}$ to represent the Cournot competition game with the restricted strategy space $[0,1)$. The following lemma, the proof of which is available in [8], bounds the market shares that solve the revenue maximization problem of each NSP, when the PDF of the users' valuation of QoS satisfies the non-increasing property.

Lemma 1. Suppose that $f(\cdot)$ is non-increasing on $[0, \beta]$. Let $\tilde{\lambda}_{i}\left(\lambda_{-i}\right)$ be a market share that maximizes the revenue of $N S P \mathcal{S}_{i}$ provided that $N S P \mathcal{S}_{-i}$ chooses $\lambda_{-i} \in[0,1)$, i.e., $\tilde{\lambda}_{i}\left(\lambda_{-i}\right) \in \arg \max _{\lambda_{i} \in[0,1)} R_{i}\left(\lambda_{i}, \lambda_{-i}\right)$. Then $\tilde{\lambda}_{i}\left(\lambda_{-i}\right) \in$ $(0,1 / 2]$ for all $\lambda_{-i} \in[0,1)$, for all $i=1,2$. Moreover, $\tilde{\lambda}_{i}\left(\lambda_{-i}\right) \neq 1 / 2$ if $\lambda_{-i}>0$, for $i=1,2$.

Lemma 1 implies that, when the strategy space is specified as $[0,1)$ and $f(\cdot)$ satisfies the non-increasing property, strategies $\lambda_{i} \in\{0\} \cup(1 / 2,1)$ is strictly dominated for $i=1,2 .{ }^{10}$ Hence, if a $\mathrm{NE}\left(\lambda_{1}^{* *}, \lambda_{2}^{* *}\right)$ of $\tilde{\mathcal{G}}_{C}$ exists, then it must satisfy $\left(\lambda_{1}^{* *}, \lambda_{2}^{* *}\right) \in(0,1 / 2)^{2}$, which yields positive revenues for both NSPs. Furthermore, since a revenue-maximizing NSP

${ }^{9} \lambda_{i} \in[0,1]$ is a weakly dominated strategy for $\mathrm{NSP} \mathcal{S}_{i}$ in $\mathcal{G}_{C}$ if there exists another strategy $\lambda_{i}^{\prime} \in[0,1]$ such that $R_{i}\left(\lambda_{i}, \lambda_{-i}\right) \leq R_{i}\left(\lambda_{i}^{\prime}, \lambda_{-i}\right)$ for all $\lambda_{-i} \in[0,1]$.

${ }^{10} \lambda_{i} \in[0,1)$ is a strictly dominated strategy for NSP $\mathcal{S}_{i}$ in $\tilde{\mathcal{G}}_{C}$ if there exists another strategy $\lambda_{i}^{\prime} \in[0,1)$ such that $R_{i}\left(\lambda_{i}, \lambda_{-i}\right)<R_{i}\left(\lambda_{i}^{\prime}, \lambda_{-i}\right)$ for all $\lambda_{-i} \in[0,1)$. 
never uses a strictly dominated strategy, the set of $\mathrm{NE}$ of $\tilde{\mathcal{G}}_{C}$ is not affected by restricting the strategy space to $[0,1 / 2]$. Based on the discussion so far, we can provide a sufficient condition on $f(\cdot)$ and $g(\cdot)$ that guarantees the existence of a NE of $\tilde{\mathcal{G}}_{C}$. The proof can be found in [8].

THEOREM 2. Suppose that $f(\cdot)$ is non-increasing and continuously differentiable on $[0, \beta] .{ }^{11}$ If $f(\cdot)$ and $g(\cdot)$ satisfy (18) and (19) (shown on the top of the next page), for all $\left(\lambda_{1}, \lambda_{2}\right) \in[0,1 / 2]^{2}$, then the game $\tilde{\mathcal{G}}_{C}$ has at least one $N E$.

We can interpret the conditions (18) and (19) as providing upper bounds for $-g^{\prime}\left(\lambda_{2}\right) / g\left(\lambda_{2}\right)$ that are determined by $f(\cdot){ }^{12}$ When the users' valuation of QoS is uniformly distributed, the conditions (18) and (19) coincide and reduce to $g\left(\lambda_{2}\right)+\lambda_{2} g^{\prime}\left(\lambda_{2}\right) \geq 0$, and thus we obtain the following corollary.

Corollary 1. Suppose that the users' valuation of QoS is uniformly distributed, i.e., $f(\alpha)=1 / \beta$ for $\alpha \in[0, \beta]$. If $g\left(\lambda_{2}\right)+\lambda_{2} g^{\prime}\left(\lambda_{2}\right) \geq 0$ for all $\lambda_{2} \in[0,1 / 2]$, then the game $\tilde{\mathcal{G}}_{C}$ has at least one $N E$.

Corollary 1 states that if the elasticity of the QoS provided by NSP $\mathcal{S}_{2}$ with respect to the fraction of its subscribers is no larger than 1 (i.e., $-\left[g^{\prime}\left(\lambda_{2}\right) \lambda_{2} / g\left(\lambda_{2}\right)\right] \leq 1$ ), the Cournot competition game with the strategy space $[0,1)$ has at least one NE. As mentioned before, this condition is analogous to the sufficient conditions for convergence in that it requires that the QoS provided by NSP $\mathcal{S}_{2}$ cannot degrade too fast with respect to the fraction of subscribers.

We briefly discuss an iterative process to reach a NE of the Cournot competition game. Theorem 2 is based on the fact that the Cournot competition game with the strategy space $[0,1 / 2]$ can be transformed to a supermodular game [10] when (18) and (19) are satisfied. It is known that the largest and the smallest NE of a supermodular game can be obtained by iterated strict dominance, which uses the best response. However, a detailed analysis of this process requires an explicit expression of the best response correspondence of each NSP, which is not readily available without specific assumptions on $f(\cdot)$ and $g(\cdot)$. Finally, we mention that the existence result of NE, although important in its own right, is only the first step toward understanding competition between the two NSPs. Subsequent issues, including the uniqueness of NE and the effects of $f(\cdot)$ and $g(\cdot)$ on NE, are left for our future work.

\section{ILLUSTRATIVE EXAMPLE}

In this section, we apply the analysis to an illustrative communications market with two NSPs. To facilitate the illustration, for NSP $\mathcal{S}_{2}$, we consider linearly-degrading QoS functions for NSP $\mathcal{S}_{2}[2]$. In the considered example, we have $q_{1}=2, g\left(\lambda_{2}\right)=1-c \lambda_{2}$ for $\lambda_{2} \in[0,1]$, where $c \in[0,1)$ is constant, and $f(\alpha)=1$ for $\alpha \in[0,1]$. Note that, for

\footnotetext{
${ }^{11}$ We define the derivative of $f(\cdot)$ at 0 and $\beta$ using a one-sided limit as in footnote 3 .

${ }^{12}$ In fact, the result of Theorem 2 also holds when the directions of the inequalities in (18) and (19) are opposite. However, we choose the directions as in (18) and (19) in order to provide an analogous interpretation to that of the sufficient condition (11) for convergence.
}

$g\left(\lambda_{2}\right)=1-c \lambda_{2}$, a larger value of the QoS degradation rate $c$ means a worse technology in terms of QoS provisioning [2].

The convergence of the considered user subscription dynamics is shown in Fig. 2(a) for a particular price pair. Starting from different initial points, Figs. 2(b) and 2(c) show the iterations of revenues and market shares, respectively, when both NSPs $\mathcal{S}_{1}$ and $\mathcal{S}_{2}$ update their market shares by using their best responses to the market share of the other NSP in the previous period. ${ }^{13}$ Since the considered QoS function satisfies Corollary 1, the Cournot competition game with the strategy space $[0,1 / 2]$ can be transformed into a supermodular game and hence the best-response dynamics is known to converge to a pure NE [10], as verified in Fig. 2 (c). It is interesting to see that all the iterations converge to the same point, suggesting that there exists a unique NE of the Cournot competition game $\tilde{\mathcal{G}}_{C}$ in the considered example. Moreover, Fig. 2(c) is consistent with Lemma 1 as the best-response market shares of both NSPs do not exceed $1 / 2$. It can also be observed from Figs. 2(b) and 2(c) that if NSP $\mathcal{S}_{2}$ has a QoS function that degrades faster (shown in dashed lines), it obtains a smaller revenue, while NSP $\mathcal{S}_{1}$ obtains a higher revenue, although the QoS degradation rate $c$ has only small impacts on market shares. This is because when NSP $\mathcal{S}_{2}$ has a QoS function that degrades faster, it tries to maintain its market share by lowering its price to compensate for a lower QoS.

\section{RELATED WORKS}

Recently, communications markets have been attracting an unprecedented amount of attention from various research communities, due to their rapid expansion. For instance, without considering the interplay between different NSPs, the authors in [4] formulated a rate allocation problem by incorporating the participation of content providers into the model, and derived equilibrium prices and data rates. In [5], the authors showed that non-cooperative communications markets suffer from unfair revenue distribution among NSPs and proposed a revenue-sharing mechanism that requires cooperation among NSPs. The behavior of users and its impact on the revenue distribution, however, were not explicitly considered. [6] studied technology adoption and competition between incumbent and emerging network technologies. Nevertheless, the model characterizing the users' valuation of $\mathrm{QoS}$ is restricted to uniform distributions and the competition between NSPs was not addressed. Moreover, only constant QoS functions were considered in [6]. Another paper related to our work is [7] in which the authors examined the evolution of network sizes in wireless social community networks. A key assumption, based on which equilibrium was derived, is that a social community network provides a higher QoS to each user as the number of subscribers increases. While this assumption is valid if network coverage is the dominant factor determining the QoS or positive network externality exists, it does not capture QoS degradation due to, for instance, user traffic congestion incurred at an NSP [2][11]. To reflect the resource constraint of an NSP, it is necessary to take into account the fact that more users using the network service will degrade the QoS (i.e., negative network effects) [2].

\section{CONCLUSION}

\footnotetext{
${ }^{13}$ The best response is computed numerically.
} 


$$
\begin{gathered}
\left\{\frac{1}{f\left(F^{-1}\left(1-\lambda_{1}-\lambda_{2}\right)\right)}+\frac{\lambda_{1} f^{\prime}\left(F^{-1}\left(1-\lambda_{1}-\lambda_{2}\right)\right)}{\left[f\left(F^{-1}\left(1-\lambda_{1}-\lambda_{2}\right)\right)\right]^{3}}\right\} g\left(\lambda_{2}\right) \\
+\left\{F^{-1}\left(1-\lambda_{1}\right)-\frac{\lambda_{1}}{f\left(F^{-1}\left(1-\lambda_{1}\right)\right)}-F^{-1}\left(1-\lambda_{1}-\lambda_{2}\right)+\frac{\lambda_{1}}{f\left(F^{-1}\left(1-\lambda_{1}-\lambda_{2}\right)\right)}\right\} g^{\prime}\left(\lambda_{2}\right) \geq 0 \\
\left\{\frac{1}{f\left(F^{-1}\left(1-\lambda_{1}-\lambda_{2}\right)\right)}+\frac{\lambda_{2} f^{\prime}\left(F^{-1}\left(1-\lambda_{1}-\lambda_{2}\right)\right)}{\left[f\left(F^{-1}\left(1-\lambda_{1}-\lambda_{2}\right)\right)\right]^{3}}\right\} g\left(\lambda_{2}\right)+\frac{\lambda_{2}}{f\left(F^{-1}\left(1-\lambda_{1}-\lambda_{2}\right)\right)} g^{\prime}\left(\lambda_{2}\right) \geq 0
\end{gathered}
$$
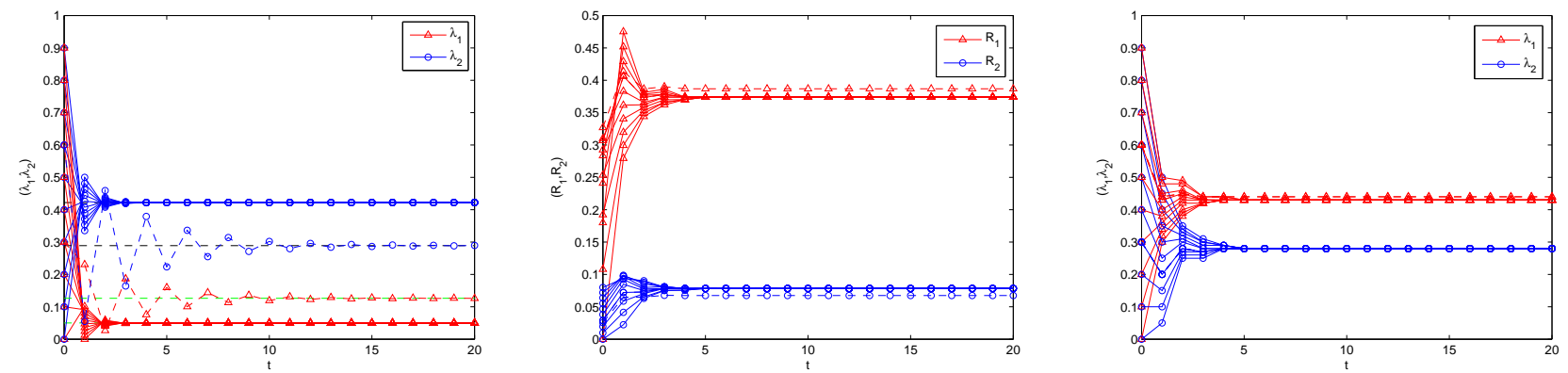

(a) Convergence of the user subscription (b) Iteration of revenues under the best- (c) Dynamics of market shares under the dynamics. $p_{1}=1.5$ and $p_{2}=0.5$. Solid: response dynamics. Solid: $g\left(\lambda_{2}\right)=1-$ best-response dynamics. Solid: $g\left(\lambda_{2}\right)=$ $g\left(\lambda_{2}\right)=1-\frac{\lambda_{2}}{8}$; dashed: $g\left(\lambda_{2}\right)=1-\frac{\lambda_{2}}{2} \cdot \frac{\lambda_{2}}{8}$; dashed: $g\left(\lambda_{2}\right)=1-\frac{\lambda_{2}}{2} . \quad 1-\frac{\lambda_{2}}{8}$; dashed: $g\left(\lambda_{2}\right)=1-\frac{\lambda_{2}}{2}$.

In this paper, we investigated the interaction between technologies, the subscription decisions of users, and the pricing strategies of NSPs in a duopoly communications market with two NSPs, each of which provides its subscribers with different levels of QoS. We constructed the dynamics of user subscription based on myopic updates, and showed that, for any prices, there exists a unique equilibrium point of the user subscription dynamics at which the number of subscribers to the two NSPs does not change. We also provided a sufficient condition on the QoS function of NSP $\mathcal{S}_{2}$ that ensures the global convergence of the user subscription dynamics. The analysis indicated that in order for the user subscription dynamics to converge, NSP $\mathcal{S}_{2}$ should select technologies in such a way that the provided QoS does not degrade too fast as more users subscribe. Then, we studied competition between the two revenue-maximizing NSPs, focusing on market share competition. We modeled the NSPs as strategic players in a non-cooperative game where each NSP aims to maximize its own revenue by choosing its market share. We obtained a sufficient condition that ensures the existence of at least one NE of the game. Finally, we illustrated our analysis with an example of a communications market, and showed that the QoS function influences the competition between the NSPs in such a way that the resource-constrained NSP $\mathcal{S}_{2}$ benefits from providing each subscriber with a higher QoS (i.e., better technology).

\section{REFERENCES}

[1] D. Acemoglu, A. Ozdaglar, and R. Srikant, "The marginal user principle for resource allocation in wireless networks," IEEE Conf. on Decision and Control, 2004.

[2] C. K. Chau, Q. Wang, and D. M. Chiu, "On the viability of Paris metro pricing for communication and service networks," IEEE Infocom, Mar. 2010.
[3] G. W. Evans and S. Honkapohja, Learning and Expectations in Macroeconomics Princeton, NJ: Princeton Univ. Press, 2001.

[4] P. Hande, M. Chiang, A. R. Calderbank, and S. Rangan, "Network pricing and rate allocation with content provider participation," IEEE Infocom, Apr. 2009.

[5] L. He and J. Walrand, "Pricing and revenue sharing strategies for Internet service providers," IEEE J. Sel. Areas Commun., vol. 24, no. 5, pp. 942-951, May 2006.

[6] Y. Jin, S. Sen, R. Guerin, K. Hosanagar, and Z.-L. Zhang, "Dynamics of competition between incumbent and emerging network technologies," NetEcon, Seattle, WA, USA, August 22, 2008.

[7] M. Manshaei, J. Freudiger, M. Felegyhazi, P. Marbach, and J. P. Hubaux, "On wireless social community networks," IEEE Infocom, Apr. 2008.

[8] S. Ren, J. Park, and M. van der Schaar, "User subscription, revenue maximization, and competition in communications markets," available at http://arxiv.org/abs/1008.5367.

[9] S. Shakkottai, R. Srikant, A. Ozdaglar and D. Acemoglu, "The price of simplicity," IEEE J. Sel. Areas in Commun., pp. 1269-1276, vol. 26, no. 7, Sep.2008.

[10] D. M. Topkis, Supermodularity and Complementarity, Princeton University Press, 1998.

[11] A. Zemlianov and G. de Veciana, "Cooperation and decision making in wireless multiprovider setting," IEEE Infocom, Mar. 2005. 